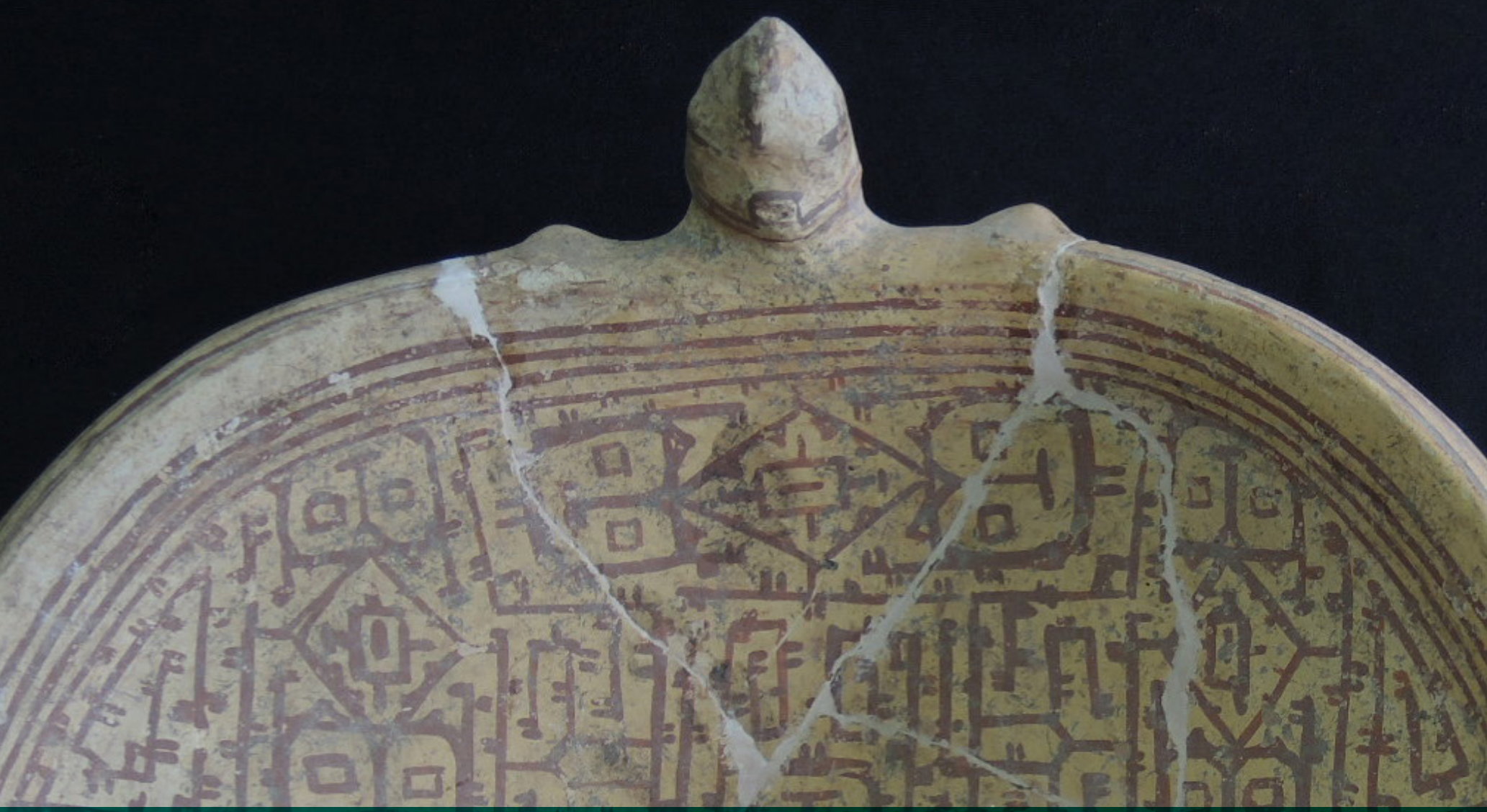

\title{
Conservar Património
}

ARP - Associação Profissional de Conservadores-Restauradores de Portugal

revista.arp.org.pt
Rua Saraiva de Carvalho ${ }^{\circ} 8$ Águas-furtadas 1250-243 Lisboa

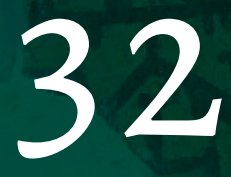

Periodicidade

Quadrimestral

Triannual

Reg. 127342
Setembro

September

2019 
Fotografia da capa Cover photograph

Maria Santana Simas, Cassia da Rosa, Cristiana Barreto,

Helena Pinto Lima, Cada instituição, um fragmento:

problemática da dispersão da coleção arqueológica marajoara

Dita Acatauassu (Amazónia, Brasil), pp. 80-87

As opiniões manifestadas na revista são da exclusiva responsabilidade dos seus autores e não traduzem necessariamente a opinião da ARP, da Direcção da revista ou do Conselho Editorial.

The opinions published in this journal are those of the authors alone and do not necessarily translate the views or opinions of ARP, the Editors of the journal or of its Editorial Board.

\section{(@) $(\Theta \Theta \Theta$}

Licenciado sob uma Licença Creative Commons

Atribuição-NãoComercial-SemDerivações 4.0 Internacional.

Para ver uma cópia desta licença, visite

http://creativecommons.org/licenses/by-nc-nd/4.o/deed.pt

This work is licensed under the Creative Commons

Attribution-NonCommercial-NoDerivatives 4.0 International License.

To view a copy of this license, visit

http://creativecommons.org/licenses/by-nc-nd/4.o/deed.en 


\section{ESTATUTO EDITORIAL}

1. A Conservar Património é uma publicação de revista online quadrimestral sem fins lucrativos, com estatuto editorial constante no $n .^{\circ} 1$, art ${ }^{\circ} 17^{\circ}$ da Lei 2/99 de 13 de Janeiro.

2. A Conservar Património é propriedade da ARP - Associação Profissional de Conservadores-restauradores de Portugal.

3. A Conservar Património é dirigida por um diretor com a colaboração de diretores-adjuntos, os quais, em conjunto, formam a direção da Revista.

4. A Conservar Património é independente do poder político, ideológico, económico ou religioso.

5. A Conservar Património pauta-se por critérios de isenção, imparcialidade, seriedade, rigor, honestidade intelectual e criatividade editorial, seguindo um compromisso de assegurar o respeito pelos princípios deontológicos e pela ética profissional.

6. A Conservar Património é uma revista de natureza técnico-científica sobre Conservação e Restauro de Bens Culturais, colaborando com outras áreas transversais das Artes, Humanidades e Ciências. Pretende proporcionar um espaço aos conservadores-restauradores para a divulgação regular dos seus estudos e atividades.

7. A Conservar Património visa:

- Constituir um repertório de informação técnica e de conteúdos especializados, numa área em que a evolução tecnológica é constante;

- Consagrar na imprensa um fórum potenciando a comunicação entre os vários intervenientes do meio empresarial, institucional e académico ligados à atividade;

- Dar destaque a conteúdos pedagógicos, dirigidos ao público em geral, tendo em vista incrementar a sensibilização para os Bens Patrimoniais;

- Promover as atividades do sector que esta representa, promover o desenvolvimento da Conservação e Restauro e da profissão de conservador-restaurador e, de uma forma geral, contribuir para a defesa e a valorização do Património Cultural.

Lisboa, 15 de Julho de 2019 


\section{CONSERVAR PATRIMÓNIO 32 \\ ARP · Associação Profissional de Conservadores Restauradores de Portugal \\ Periodicidade Quadrimestral \\ Setembro 2019 \\ revista.arp.org.pt}

\section{Editorial}

6 ELIN FIGUEIREDO, ANA CLARO, ANA ZÉLIA MILLER, SÍLVIA O. SEQUEIRA, TIAGO MIGUEL FERREIRA

Novos desafios para a Conservar Património

New challenges for Conservar Património

\section{Artigos Articles}

ANTONIO J. SÁNCHEZ FERNÁNDEZ, BEATRIZ PRADO-CAMPOS

Diseño de embalajes para objetos culturales: método de plantillas por fotogrametría

Packaging design for cultural objects: photogrammetry templates procedure

Desenho de embalagens para objectos culturais: modelos por fotogrametria

18 SÍlVIA OLIVEIRA SEQUEIRA, HUGO PAIVA DE CARVALHO, NUNO MESQUITA, ANTÓNIO PORTUGAL, MARIA FILOMENA MACEDO

Fungal stains on paper: is what you see what you get?

Manchas de fungos em papel: o que se vê é o que se obtém?

Preparing the foundation for stable gilding: the origins of materials and practices - a review Das fundações para um douramento estável: as origens dos materiais e práticas - uma revisão

Going back to collections: a study case of the Florentino Ameghino collection housed in the Museo de La Plata (Argentina)

De volta às colecções: o caso da colecção Florentino Ameghino

do Museu de La Plata (Argentina)

\section{Notas Notes}

79 MARIA SANTANA SIMAS, CASSIA DA ROSA, CRISTIANA BARRETO, HELENA PINTO LIMA

Cada instituição, um fragmento: problemática da dispersão da coleção arqueológica marajoara Dita Acatauassu (Amazónia, Brasil)

A fragment in each institution: the dispersion of Dita Acatauassu Marajoara Archaelogical Collection (Amazonia, Brazil) 
CONSERVAR PATRIMÓNIO

Revista académica com avaliação por pares

Academic peer-reviewed journal

Edição Publisher

Associação Profissional de Conservadores-Restauradores de Portugal (ARP)

Rua Saraiva de Carvalho n ${ }^{\circ} 8$ Águas-furtadas 1250-243 Lisboa, Portugal

Contribuinte VAT registration number n. ${ }^{\circ} 503602981$

Periodicidade Frequency

Quadrimestral Triannual

Registo no Instituto da Comunicação Social

124638

ISSN

2182-9942 edição digital, n. ${ }^{\circ} 17$ e seguintes

digital edition, no. 17 and following

1646-043X edição em papel, n. ${ }^{\circ}$ S 1-16

paper edition, no. 1-16

DOI

https://doi.org/10.14568/cp

Director Editor-in-chief

ELIN FIGUEIREDO

Directores-adjuntos Associate editors

ANA CLARO

ANA ZÉLIA MILLER

SÍLVIA O. SEQUEIRA

TIAGO MIGUEL FERREIRA

Paginação da revista Journal layout

JOANA \& MARIANA 


\section{Director Editor-in-chief}

ELIN FIGUEIREDO

Investigadora, Centro de Investigação de Materiais, CENIMAT/i3N, Universidade NOVA de Lisboa, Portugal

\section{Directores-adjuntos Associate editors}

ANA CLARO

Investigadora Integrada, CHAM - Centro de Humanidades, Faculdade de Ciências Sociais e Humanas, Universidade NOVA de Lisboa, Portugal ANA ZÉLIA MILLER

Investigadora Auxiliar, Laboratório HERCULES, Universidade de Évora, Portugal

SÍLVIA O. SEQUEIRA

Professora Adjunta Convidada, Instituto Politécnico de Tomar, Portugal Investigadora, VICARTE, FCT - Universidade NOVA de Lisboa, Portugal TIAGO MIGUEL FERREIRA

Investigador, Institute for Sustainability and Innovation in Structural Engineering (ISISE), Universidade do Minho, Portugal Professor Auxiliar Convidado, Universidade de Coimbra, Portugal

\section{Comissão de redacção Copy editing board}

RUI MAIO

Investigador, RIscos e Sustentabilidade na COnstrução (RISCO), Universidade de Aveiro, Portugal

JOÃO LUÍS ANTUNES

Químico, Portugal

GUILHERME PINHEIRO POZZER

Investigador, Centro Interdisciplinar de Ciências Sociais

(CICS.NOVA.UMinho), Universidade do Minho, Portugal

THIAGO SEVILHANO PUGLIERI

Professor Auxiliar, Departamento de Museologia,

Conservação e Restauro, Universidade Federal de Pelotas, Brasil

\section{Conselho editorial Editorial board \\ CHRISTIAN DEGRIGNY}

Conservation Scientist, Haute École de Conservation-Restauration Arc, La Chaux-de-Fonds, Suisse

M. a JOSÉ GONZÁLEZ LÓPEZ

Profesora Titular, Departamento de Pintura,

Facultad de Bellas Artes, Universidad de Sevilla, España

MÁRIO MENDONÇA DE OLIVEIRA

Professor, Universidade Federal da Bahia, Brasil

ROSÁRIO VEIGA

Investigadora Principal com Habilitação para Coordenação Científica, Laboratório Nacional de Engenharia Civil, Lisboa, Portugal

SALVADOR MUÑOZ VIÑAS

Profesor, Universitat Politècnica de València, España

VÍTOR SERRÃO

Professor Catedrático, Instituto de História da Arte,

Faculdade de Letras da Universidade de Lisboa, Portugal 
A revista está indexada ou referenciada nas seguintes bases de dados bibliográficas internacionais: The journal is abstracted or indexed in the following international bibliographic databases:

- AATA - Abstracts of International Conservation Literature, Getty Conservation Institute, http://aata.getty.edu

- BCIN - The Bibliographic Database of the Conservation Information Network, Canadian Heritage Information Network, http://www.bcin.ca

- Chemical Abstracts, American Chemical Society, http://www.cas.org

- CIRC - Clasificación Integrada de Revistas Científicas, EC3METRICS, https://clasificacioncirc.es

- Crossref, http://www.crossref.org

- CWTS Journal Indicators, Leiden University, http://www.journalindicators.com

- DOAJ - Directory of Open Access Journals, http://www.doaj.org

- EBSCO Art Source, https://www.ebscohost.com

- ERIH PLUS - European Reference Index for the Humanities and the Social Sciences, Norwegian Social Science Data Services, https://dbh.nsd.uib.no/publiseringskanaler/erihplus/

- Google Scholar, http://scholar.google.com

- Index Copernicus Journals Master List, http://journals.indexcopernicus.com

- Ingenta Connect, http://www.ingentaconnect.com

- Latindex - Sistema Regional de Información en Línea para Revistas Científicas de América Latina, el Caribe, España y Portugal, http://www.latindex.unam.mx

- MIAR - Matriz de Información para el Análisis de Revistas, Universitat de Barcelona, http://miar.ub.edu

- Microsoft Academic, https://academic.microsoft.com

- OpenAIRE - Open Access Infrastructure for Research in Europe, https://explore.openaire.eu

- REDIB - Red Iberoamericana de Innovación y Conocimiento Científico, http://www.redib.org

- Scimago, http://www.scimagojr.com

- Scopus, Elsevier, https://www.scopus.com

- Web of Science - Emerging Sources Citation Index (ESCI), Clarivate Analytics, https://webofknowledge.com 